\title{
A NOTE ON THE GENUS OF A KNOT
}

\section{S. KINOSHITA ${ }^{1}$}

For each polygonal knot $k$ in a 3 -sphere $S^{3}$ there exists a polyhedral orientable surface $M_{k}$ whose boundary is $k([1 ; 2])$. The genus of such a knot $k$, denoted by $g(k)$ below, was defined by H. Seifert [2] as the minimal number of genera of all such $M_{k}$. Now let us define $\tilde{g}(k)$ for each polygonal knot $k$ as the minimal number of genera of all orientable surfaces $M_{k}$ whose boundaries are $k$, where $M_{k}$ may be wildly imbedded. Is $\tilde{g}(k)=g(k)$ ? This is a problem proposed by R. H. Fox. The purpose of this short note is to prove that the equality holds.

Proof. Let $k$ be a polygonal knot in $S^{3}$. Let $M$ be a polyhedral orientable surface and $h$ a homeomorphism of $M$ in $S^{3}$ such that $h(\partial M)=k$. Then we are only to prove that $g(M)$, the genus of $M$, is equal to or greater than $g(k)$.

Let $T$ be a tubular neighborhood of $k$. Bing's approximation theorem [3] shows that for each $\epsilon>0$ there exists a semilinear homeomorphism $h^{\prime}$ of $M$ into $S^{3}$ such that for each $x \in M d\left(h(x), h^{\prime}(x)\right)<\epsilon$. If we choose $\epsilon$ as to be sufficiently small, we may suppose that $h^{\prime}(\partial M)$ $=k^{\prime}$ is contained in $T$ and that $k$ is a companion of $k^{\prime}$ with the winding number one in the sense of $H$. Schubert [4]. By definition $g\left(h^{\prime}(M)\right) \geqq g\left(k^{\prime}\right)$. Further H. Schubert [4] shows that if $k$ is a companion of $k^{\prime \prime}$ with the winding number $\alpha$, then $g\left(k^{\prime \prime}\right) \geqq \alpha g(k)+g\left(k^{*}\right)$, where $k^{*}$ is a suitably defined polygonal knot. Thus $g\left(k^{\prime}\right) \geqq g(k)$ in our case. Therefore $g(M)=g\left(h^{\prime}(M)\right) \geqq g\left(k^{\prime}\right) \geqq g(k)$, which completes the proof.

\section{REFERENCES}

1. F. Frankl and L. Pontrjagin, Ein Knotensatz mit Anwendung auf die Dimensionstheorie, Math. Ann. 102 (1930), 785-789.

2. H. Seifert, Über das Geschlecht von Knoten, Math. Ann. 110 (1934), 571-592.

3. R. H. Bing, An alternative proof that 3-manifolds can be triangulated, Ann. of Math. 69 (1959), 37-65.

4. H. Schubert, Knoten und Vollringe, Acta Math. 90 (1953), 131-286.

InSTITUTE for AdVANCEd Study AND OSAKA UNIVERSITY

Received by the editors May 1, 1961.

1 Supported by National Science Foundation grant G14779. 